\title{
Diamondoids - The Molecular Lego of Biomedicine, Materials Science and Nanotechnology
}

\section{Mansoori G Ali*}

Department of Bioengineering, University of Illinois at Chicago, Chicago, USA

\section{A Brief Description of Diamondoids}

Diamondoids are a peculiar class of organic molecules with unique structures and properties. Diamondoid molecules (AKA polymantanes, adamantologs) are cage-like, ultra stable, saturated hydrocarbons. The basic repetitive unit of diamondoids is a ten-carbon tetracyclic cage system called "adamantane" (Figure 1a). Adamantane consists of cyclohexane rings in 'chair' conformation. These molecules are called "diamondoids" because their carbon-carbon framework is completely, or largely, superimposable on the diamond lattice (Figure 2). Diamondoids show unique properties due to their exceptional atomic arrangements. The discovery of adamantane cage in 1933 [1] and its direct synthesis in 1957 [2] has turned this diamondoid and its derivatives into readily available compounds with numerous applications. Since 1960, the interest in practical applications of diamondoid molecules has steadily increased [3]. Diamondoids are presently molecular building blocks for biomedicine, materials science and nanotechnology that enable the design and manufacturing of nanometer-scale structures programmed to have virtually any desired shape and properties [4-6].

Diamondoids were first discovered in petroleum [1] and since there has been limited progress in synthesizing them there have been continuous efforts in exploring them in petroleum and other fossil fuels [7-10]. The presence of diamondoids in fossil fuels has become much more than a chemical curiosity and has advanced to be a resourceful instrument in biomedicine, materials science, and nanotechnology [6].

It has also been of major interest to discover the tremendous ways of derivativizing these molecules to do wonders in biomedicine, materials science and in the emerging field of nanotechnology [4-6]. The presence of chirality is an important feature in many diamondoids. The vast number of structural isomers and stereoisomers is another property of diamondoids. For instance, octamantane possesses hundreds of isomers in five molecular weight classes. The octamantane class with

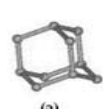

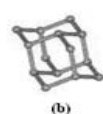
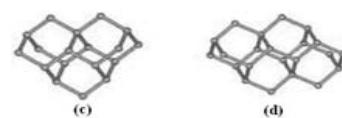

Figure 1: Molecular structures of lower diamondoids: (a): Adamantane (b): Diamantane (c): Triamantane (d): Anti- isomer of Tetramantane.

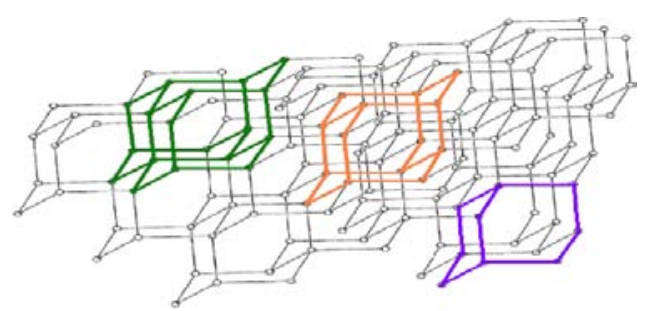

Figure 2: Structures of adamantane, diamantane and triamantane superimposed over the three-dimensional diamond network. formula $\mathrm{C}_{34} \mathrm{H}_{38}$ and molecular weight 446 has 18 chiral and achiral isomeric structures. Furthermore, there is unique and great geometric diversity within these isomers. For example, rod-shaped diamondoids (with the shortest one being $1.0 \mathrm{~nm}$ long) and disc-shaped and screwshaped diamondoids (with different helical pitches and diameters) have been recognized [11]. For diamondoids and derivatives property predictions and characterizations in addition to variety of experimental techniques which are reported in [6] the quantum mechanical abinito methods are being utilized [12-16].

Diamondoid-based derivatives are used to fight cancer, Alzheimer's disease as well as viral, bacterial, and parasital infectious diseases. Utilization of diamondoids in synthesis of high temperature polymers, in polymer nanocomposites, and in crystal engineering are some of their major applications in materials science. Due to their six or more linking groups (Figure 3 ), diamondoids and derivative family of compounds (with over 20,000 variants) are the best candidates for templates and molecular building blocks in nanotechnology such as molecular self-assmbly (Figure 4), in design of NEMS and MEMS, in nanodrug delivery and targeting, in DNA-directed assembly nanostructure formation (Figure 5), and in host-guest chemistry.

Diamondoids have very high melting points as compared with other hydrocarbons and organic molecules. Diamondoids have negative electron affinities and they are transparent to visible light and high electrical insulating properties as diamond. Since diamondoids

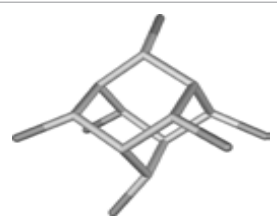

Figure 3: Six linking groups of adamantine [17]
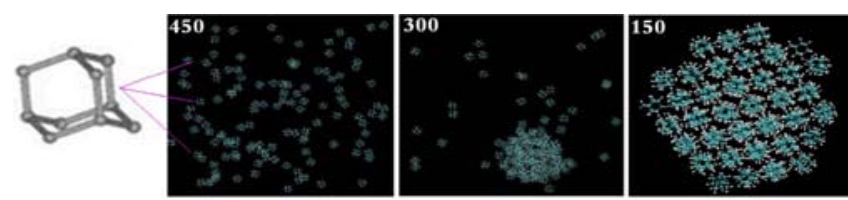

Figure 4: Self-assembly snapshots of 125 molecules of the adamantane, as temperature, in degrees Kelvin, is decreased [18,19].

*Corresponding author: Mansoori G Ali, Department of Bioengineering, University of Illinois at Chicago, Chicago, USA, E-mail: mansoori@uic.edu

Received May 02, 2013; Accepted May 10, 2013; Published May 15, 2013

Citation: Mansoori GA (2013) Diamondoids -The Molecular Lego of Biomedicine, Materials Science and Nanotechnology. J Bioanal Biomed 5: e116. doi:10.4172/1948-593X.1000e116

Copyright: @ 2013 Mansoori GA. This is an open-access article distributed under the terms of the Creative Commons Attribution License, which permits unrestricted use, distribution, and reproduction in any medium, provided the original author and source are credited. 

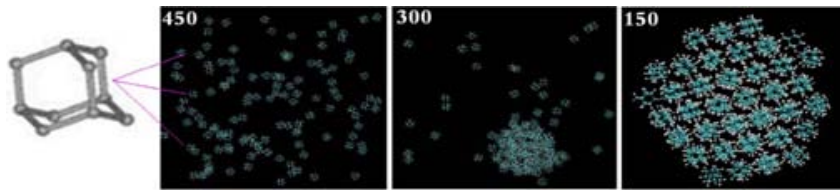

Figure 5: Diamondoid-DNA cube-shaped nanostructured assembly: (i) Green dotted lines showing hydrogen bonding between complementary sequences. (ii) Electrostatic potential surface of the cube-shaped nanostructure, where the red regions bear negative partial charges whereas the positive sites are colored in blue. (iii) The Corey-Pauling-Koltun model of the same nanostructure reflecting the square shape of each face $[20,21]$.

also possess low strain energy, they are more stable and stiff, resembling diamond in a broad sense. They possess superior strength-to-weight ratio.

\section{Biomedical Applications of Diamondoids and Derivatives}

There have been great research interest and activities in understanding how diamondoid molecules interact with living systems, how these interactions could be useful in their biomedical applications, and characteristics of these molecules as tools for diagnosis and cell metabolism screening.

Among all diamondoids, adamantane and its derivatives have been most widely used for drug design.Starting from the late 50's and early 60 's, the hydrophob ic nature associated with the compact and highly symmetrical structure of the adamantane molecule have attracted researches interested in the discovery of structurally unprecedented drugs, such as polyhaloadamantane pesticides [22]; and new or improved biological activities conferred by a caged moiety attached to known bioactive substances, such as orally active hypoglycemic sulfonylureas [23]. It is also known that adamantly group changes the properties of known drugs or provides an important pharmacophore for the design of new drugs [24].

Diamondoid derivatives in drug delivery and drug targeting, especially for the fact that due to their lipophilicity / hydrophobicity they can easily pass through blood-brain barrier. Adamantane nucleus, which is present in all adamantane derivatives, might be responsible for their ability to penetrate into the blood-brain barrier (BBB) and their accumulation in the CNS. Their applications for pharmacophorebased drug design, for drug delivery and drug targeting systems is a new matter of considerable importance [6].

Already diamondoids are used to design drugs in fighting infectious diseases like Influenza Viruses and HIV, bacterial infections like Gram-positive bacteria and MTB (Mycobacterium tuberculosis), and parasitic infection treatments like antimalarial drugs. Fighting cancer with diamondoids derivatives is another important application of these interesting compounds. Among such derivatives adaphostin retinoid derivative, is used for chemotherapy and exert selective antiproliferative and cytodifferentiating effects. These actions are of special interest in the search for new antineoplasic agents. Neuroprotective effect of memantine (a derivative of asamantane) and some other diamondoids derivatives for Alzheimer's disease is another important set of biomedical applications of these compounds. Design of new antidotes to counteract the effect of a poison by diamondoid derivatives is an additional important application of these substances. A number of adamantane derivatives possess hypoglycemic action. And they are used as drugs fordiabetes treatments. In conclusion,

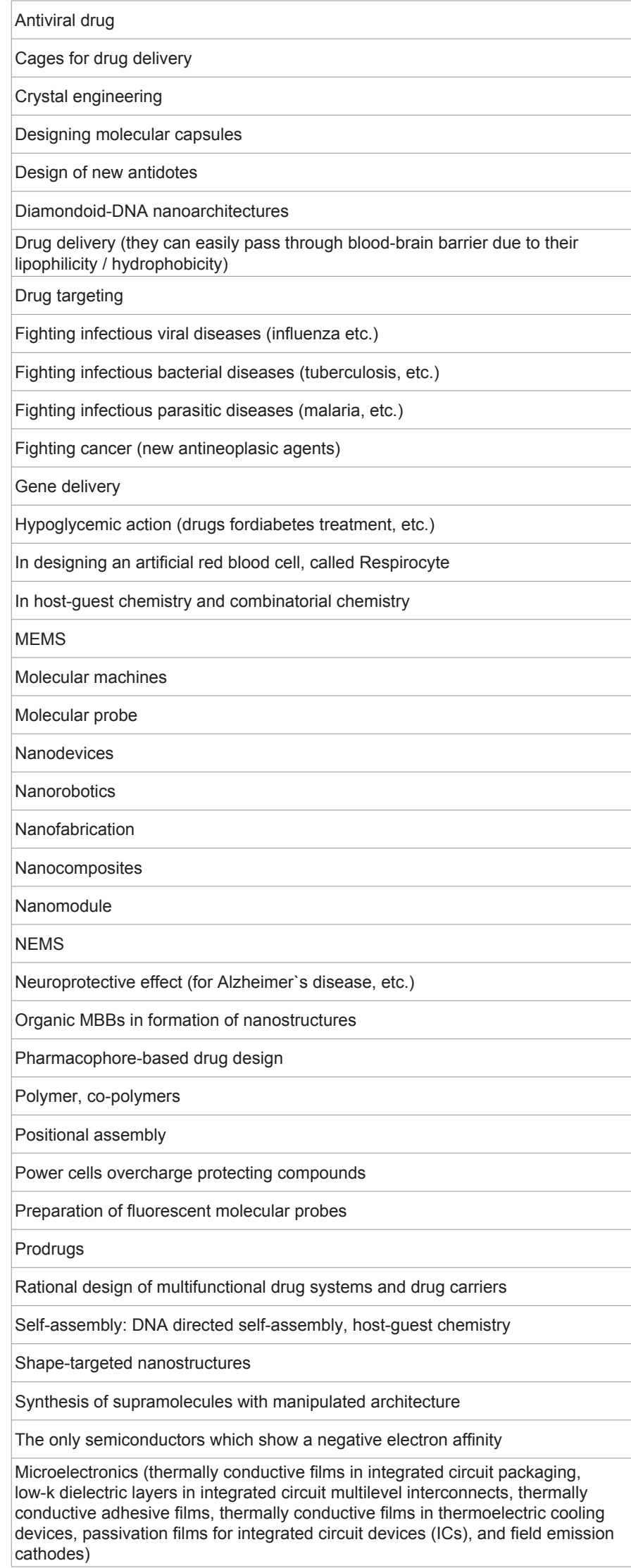

Table 1: Major Applications of Diamondoids and Derivatives. 


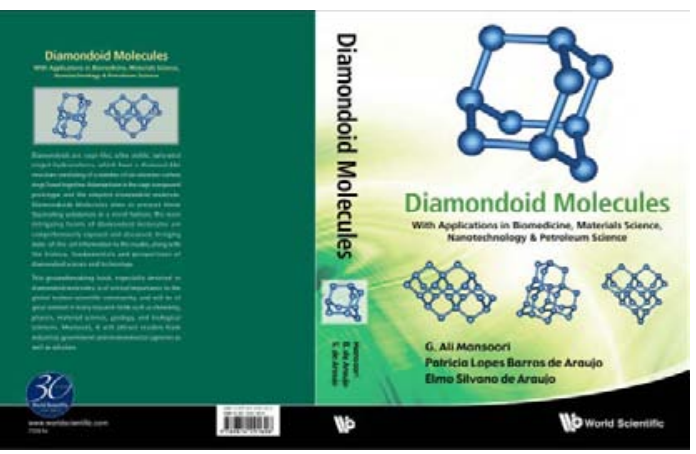

Figure 6: Diamondoid molecules with applications in biomedicine, materials science, nanotechnology and petroleum science [6].

diamondoids derivatives are an important family of compounds able to render diverse, strong, and sometimes unique biological properties to a multitude of drug classes. As many of these molecules are still in initial development phases, it will not be; surprising if a considerable amount of new cage compounds start to be a good addition to our medical supplies in a near future [6].

In Table 1 we report an alphabetical list of applications of diamondoids in biomedicine, materials science, nanotechnology. Diamondoids are fascinating molecules with interesting and promising applications as molecular lego in biomedicine, materials science, and nanotechnology. Recently a book (Figure 6) was published in which the details of diamondoids sources, properties and applications were reported.

\section{References}

1. Landa S, Machacek V, Mzourek M, Landa M (1933) Chim Ind (Publ. No. 506).

2. Schleyer PvR (1957) A simple preparation of adamantine. J Am Chem Soc 79: 3292-3292.

3. Mansoori GA (2007) Diamondoid molecules. Advances in Chemical Physics 136: 207-258.

4. Mansoori GA (2005) Principles of Nanotechnology (Molecular-Based Study of Condensed Matter in Small Systems). World Scientific Publishing Corporation, USA.

5. Mansoori GA, George TF, Assoufid L, Zhang G (2007) Molecular Building Blocks for nanotechnology-from diamondoids to nanoscale materials and applications. Topics in Applied Physics 109: 426 pages Springer: New York, USA.

6. Mansoori GA, De Araujo PLB, De Araujo ES (2012) Diamondoid molecules: with applications in biomedicine, materials science, nanotechnology and petroleum science. Word Scientific, Hackensack, USA.

7. Vakili Nezhaad GR, Soltani B, Mansoori GA, Kiani-Nassab K (2005) Investigation of the existence of diamondoids in South-West Iranian crude oils. IJNN 1: 65-74.

8. Mansoori GA (2009) A unified perspective on the phase behaviour of petroleum fluids. Int $\mathrm{J}$ of Oil, Gas and Coal Technology 2: 141-167.

9. Mansoori GA (2010) ELMI a Sarlar, J. Reservoir and Petroleum Engineering SOCAR, pp.12-23.
10. De Araujo PLB, Mansoori GA, De Araujo ES (2012) Diamondoids: occurrence in fossil fuels, applications in petroleum exploration and fouling in petroleum production. Int J of Oil, Gas and Coal Technology 5: 316-367.

11. Dahl JE, Liu SG, Carlson RM (2003) Isolation and structure of higher diamondoids, nanometer-sized diamond molecules. Science 299: 96-99.

12. Zhang GP, George TF, Assoufid L, Mansoori GA (2007) First-principles simulation of the interaction between adamantane and an atomic-forcemicroscope tip. Phys Rev B 75: 035413.

13. Xue Y, Mansoori GA (2008) Quantum conductance and electronic properties of lower diamondoid molecules and derivatives. International Journal of Nanoscience 7: 63.

14. Marsusi F, Mirabbaszadeh K, Mansoori GA (2009) Opto-electronic properties of adamantane and hydrogen-terminated sila- and germa-adamantane: A comparative study. Physica E: Low-dimensional Systems and Nanostructures 41: 1151-1156.

15. Mansoori GA, George TF, Zhang GP, Assoufid L (2009) Structure and optoelectronic behavior of diamondoids with applications as MEMS and at the nanoscale level. Encyclopedia of Nanotechnology, Nova Publishers, Chapter 1: $527-545$.

16. Araujo ESD, Mansoori GA, Xue Y, Lopes Barros de Araujo P (2011) Diamondoid molecules behavior prediction by ab initio methods. Physics Express 1: 67-88.

17. Ramezani H, Mansoori GA (2007) Diamondoids as molecular building blocks for nanotechnology. Molecular Building Blocks for Nanotechnology: Topics in Applied Physics 109: 44-71.

18. Xue Y, Mansoori GA (2010) Self-assembly of diamondoid molecules and derivatives (MD simulations and DFT calculations). Int J Mol Sci 11: 288-303.

19. Xue Y, Mansoori GA (2010) Phase transition and self-assembly of lower diamondoids and derivatives. MRS Proceedings Vol 1282.

20. Ramezani H, Mansoori GA, Saberi MR (2007) Diamondoids-DNA nanoarchitecture: from nanomodules design to self-assembly. Journal of Computational and Theoretical Nanoscience 4: 96-106.

21. Ramezani H, Saberi MR, Mansoori GA (2007) Diamondoids and DNA Nanotechnologies. IJNN 3: 24-35.

22. Webber WC, Harthoorn PA (1959) Chlorinated and brominated polycyclic hydrocarbons, their preparation, and insecticidal compositions containing them. United Kingdom Patent GB819240.

23. Gerzon K, Krumkalns EV, Brindle RL, Marshall FJ, Root MA (1963) The adamantyl group in medicinal agents. I. hypoglycemic n-arylsulfonyl-n -adamantylureas. J Med Chem 6: 760-763.

24. Lamoureux G, Artavia G (2010) Use of the adamantane structure in medicinal chemistry. Curr Med Chem 17: 2967-2978. 